\title{
Wireless Multi Sensor Bracelet with Discreet Feedback
}

\author{
Martin Ouwerkerk \\ Philips Group Innovation \\ High Tech Campus \\ Eindhoven, Netherlands \\ +31402742913 \\ martin.ouwerkerk@philips.com \\ Rafal Kocielnik \\ Mathematics and Computer Science \\ Eindhoven University of Technology, Den Dolech 2, \\ 5612 AZ Eindhoven, Netherlands \\ +31402474935 \\ r.d.kocielnik@tue.nl \\ Henk Huijgen \\ Philips Group Innovation \\ High Tech Campus \\ Eindhoven, Netherlands \\ +31402797307 \\ henk.huijgen@philips.com
}

\author{
Pierre Dandine \\ Electrical Engineering \\ INSA \\ Lyon, France \\ +33647927699 \\ pierre.dandine@insa-lyon.fr
}

\author{
Dennis Bolio \\ Philips Group Innovation \\ High Tech Campus \\ Eindhoven, Netherlands \\ +31652788395 \\ dennis.bolio@philips.com
}

\author{
Johanna Mercurio \\ Mobile Life Centre at SICS \\ Swedish ICT AB, Box 1263 \\ 16429 Kista Sweden \\ +46702262399 \\ johmerc@sics.se \\ Joyce Westerink \\ Philips Group Innovation \\ High Tech Campus \\ Eindhoven, Netherlands \\ +31631628153 \\ joyce.westerink@philips.com
}

\begin{abstract}
A novel wireless multi sensor bracelet has been developed. The design choices of the bracelet - based on insights obtained with a predecessor sensor bracelet -, as well as the rationale for the choice of sensors, are presented. The hardware and software architecture are described. An example of obtained sensor data is shown. The limited battery life of the performance optimized product software fell short of the one week design target. A power optimization of the software has been made, which meets the battery life design target. It is based on current consumption measurements, and optimized sensor timing. The tradeoffs between high performance - short battery life, and low performance - long battery life are analyzed. The learnings from recent field studies on work-related stress and affective health are discussed.
\end{abstract}

\section{Categories and Subject Descriptors}

H.1.2 User/Machine Systems J.3 Life and Medical Sciences Health

\section{General Terms}

Performance, Design, Reliability, Experimentation, Human Factors, Verification

\section{Keywords}

Ambulatory sensing, unobtrusive, stress, low power technology.

\section{INTRODUCTION}

Unobtrusive sensing of emotional arousal in a daily life setting provides insight into what triggers a person, and which settings or behavior causes most stress. Such information can only be acquired when the monitoring is done almost continuously. A sensor bracelet is developed which is comfortable to wear, acceptable to family, friends, or colleges, and capable of wireless streaming of measured sensor data, as well as embedded computation allowing the assessment of emotional arousal peaks. Based on such assessment a discreet warning may be given to the wearer in the form of a vibration of the device.

As part of an Emotion Sensing Platform in 2008 a skin conductance wristband, the Discreet Tension Indicator (DTI-1) was made [1]. It featured embedded assessment of the measured skin conductance, allowing it to detect episodes of high arousal. A built-in vibration motor can signal this to the wearer in a discreet fashion. Figure 1 shows a picture of the DTI-1 skin conductance wristband.

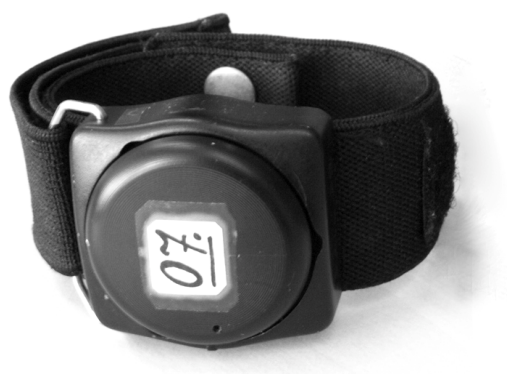

Figure 1 Discreet Tension Indicator sensor wristband DTI-1 In a book chapter of the Philips Research Book series the technical aspects of the DTI-1 are described in detail [2]. The DTI-1 was used in a pilot study on pre-tournament stress development of elite swimmers. In another pilot study the development of aggression was monitored in a crisis ward of a mental hospital [3].

In an attempt to develop a consumer lifestyle product utilizing skin conductance a bracelet prototype was developed for an 
internal venture. Details on this work are to be published in a book chapter [4]. In parallel the electronics of the DTI-1 were used in a skin conductance wristband for stock traders, called the Rationalizer [5]. All the above work was done within Philips.

Outside Philips the group of Rosalind Picard at MIT developed a skin conductance wristband for sensing of sympathetic nervous system activation [6]. Based on this work a commercial version was offered until recently by a company called Affectiva [7].

In the framework of research projects sponsored by EIT ICT Labs that aimed to measure stress in daily life situations funding became available to develop a successor of the DTI-1. This paper describes all aspects of this new sensor wristband, the DTI-2 (see Figure 2).

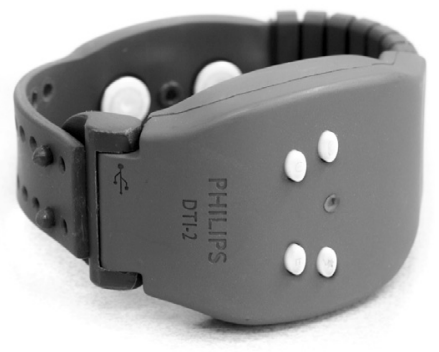

Figure 2 Novel wireless multi sensor bracelet DTI-2

\section{DESIGN ASPECTS}

The Discreet Tension Indicator DTI-2 is a sensor wristband, which offers a number of sensing modalities. The wristband is designed to be worn by participants in studies and trials.

Experience with ambulatory skin conductance monitoring was acquired with the DTI-1 wristband. A number of shortcomings were identified based on issues that came up in the field trials. In Table 1 these issues are listed along with changes implemented in the DTI-2.

A skin temperature sensor was added to the DTI-2 to complement on information of the user state. For example, Lundqvist et al. [17] found that skin temperature might be linked to hedonic tone. The skin temperature sensor further allows triangulation i.e., to use multiple sensor data to describe a user state or to describe a user state with a higher robustness. There were additional user requests which were not implemented in the DTI-2, such as location awareness (GPS), microphone, heart rate measurement at the wrist, Wi-Fi connectivity. In most cases these requests were making it impossible to keep the dimensions, the weight and the battery life within a useful range.

\subsection{Intended use}

The DTI-2 device is to be used to monitor at the left or right wrist of humans: the skin conductance, skin temperature, ambient temperature, ambient light level, and 3-dimensional acceleration. The sensor data can be streamed live to a receiving station via a Bluetooth wireless link. The device is not to be used as a medical device. Hence no diagnosis or treatment of a medical illness can or may be performed based on the data obtained by this device.
Table 1. Issues in DTI-1 with solutions in DTI-2 wristband

\begin{tabular}{|c|c|}
\hline DTI-1 issue & DTI-2 solution \\
\hline Zigbee radio dongle needed & $\begin{array}{l}\text { Bluetooth transceiver instead of } \\
\text { Zigbee }\end{array}$ \\
\hline Max 25 hours data storage & $\begin{array}{l}2 \mathrm{~GB} \text { microSD card for weeks } \\
\text { of storage }\end{array}$ \\
\hline $\begin{array}{l}\text { Complicated data readout } \\
\text { procedure }\end{array}$ & $\begin{array}{l}\text { Easy data readout via USB to } \\
\text { any computer }\end{array}$ \\
\hline Max 8 hours battery life & $\begin{array}{l}\text { Over one week battery life } \\
\text { (without radio) }\end{array}$ \\
\hline $\begin{array}{l}\text { Coolflux DSP has limited } \\
\text { availability }\end{array}$ & $\begin{array}{l}\text { MSP430 controller is in } \\
\text { widespread use }\end{array}$ \\
\hline No user interface & $\begin{array}{l}\text { Event marker, } 2 \text { LED arrays for } \\
\text { user info }\end{array}$ \\
\hline $\begin{array}{l}\text { Vibration motor buzzer on } \\
\text { outside }\end{array}$ & $\begin{array}{l}\text { Vibration motor buzzer inside } \\
\text { casing }\end{array}$ \\
\hline No indicator for radio link & $\begin{array}{l}\text { Blue LED signals Bluetooth } \\
\text { activity }\end{array}$ \\
\hline No indicator for low battery & $\begin{array}{l}\text { Battery voltage shown via LED } \\
\text { arrays }\end{array}$ \\
\hline No error handling & $\begin{array}{l}\text { Error messages via LED arrays } \\
\text { and in } \log \text { file }\end{array}$ \\
\hline No info on context of data & $\begin{array}{l}\text { Light and device temperature- } \\
\text { sensor offer context info }\end{array}$ \\
\hline $\begin{array}{ll}\text { Complicated } & \text { battery } \\
\text { charging procedure } & \end{array}$ & $\begin{array}{l}\text { Battery charging via USB } \\
\text { connector }\end{array}$ \\
\hline No date-time awareness & $\begin{array}{l}\text { Date/time clock even in off } \\
\text { mode }\end{array}$ \\
\hline
\end{tabular}

\subsection{Application requirements}

The intended use leads to the following application requirements:

\section{Physical requirements}

- Dimensions, weight, and shape of the device optimized for ambulatory use.

- Robust casing

- Comfortable fit to wide range of wrist sizes

- Neutral appearance, to avoid drawing attention to the device

\section{Sensor requirements}

- Measure the skin conductance at the underside of the wrist

- Measure the 3-D acceleration of the device

- Measure the ambient light level

- Measure the temperature of the device

- Measure the battery voltage at $2 \mathrm{~Hz}$ or less

- Date/time awareness with millisecond precision

- Data collection without maintenance for at least one week

- Built-in storage for several weeks of data at highest sampling rate

\section{User interface requirements}

- Event marker with millisecond precision

- User interface for feedback and warning signals

- On/off switching of device 
- On/off switching of transceiver

- Wireless connection for real-time streaming of data to a receiving station without dongle

- Fast USB connection to allow transfer of large amounts of data

\section{Maintenance requirements}

- Easy recharging of the battery in less than 2 hours

- Provide a means to update the firmware

- Provide a means to replace the battery

- Provide a means to replace the data storage card

- Error logging

\section{Regulatory requirements}

- Block USB-connector while the device is on the wrist

- Unique serial number

- User manual for end-user in language of end-user

- Documentation of all design and manufacturing information of the device

- Compliant to safety standard EN 60950-1:2006/A 1:2009/A 1:2010/A12:2011

- Compliant to EN 301 489-1 V1.8.1, EN 301 489-17 V2.1.1 and EN 300328 V1.7.1

\subsection{Implementation}

The dimensions of the DTI-2 casing are $39.5 \times 12 \times 63.5 \mathrm{~mm}$, which is large enough to contain the battery, the electronics main board, and the user interface board. The strap is segmented. To enable the positioning of the skin conductance electrodes at the underside of the wrist such that one electrode is at the center, and the other is at the side of the thumb, an appropriate number of segments can be removed (see Figure 2).

\subsection{Electronics}

The electronics consists of four interconnected printed circuit boards (PCBs): the user interface board, the sensor board, the main board, plus a patch board to allow battery charging via the USB connector.

In the block diagram shown in Figure 3 the PCBs are shown as light blue rectangles, the patch board, which connects the $+5 \mathrm{~V}$ USB pin to the battery, is omitted.

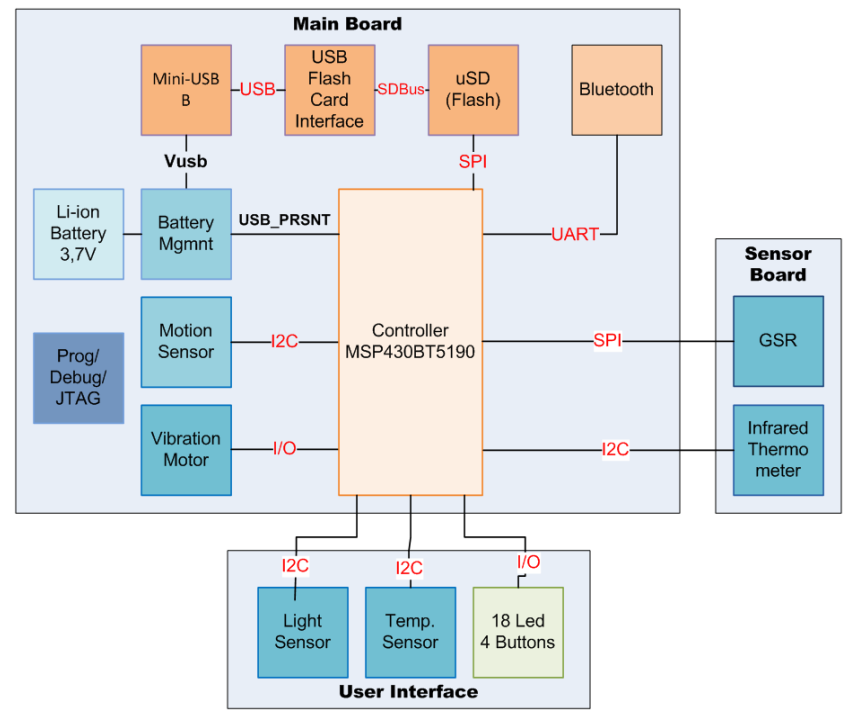

Figure 3 Electronics diagram of the DTI-2 sensor wristband

\subsubsection{Flexible main printed circuit board}

The casing of the DTI-2 has a shape that curves around the wrist (see Figure 2). The main PCB has been manufactured as a flex foil with two bending zones, to use the full length of the casing. The two segments at the ends of the flex are fitted with a stiffener to improve mechanical stability. The presence of the lithium polymer battery stabilizes the central part of the flex, when mounted in the casing. On one side of the flex, the USB connector is placed. The opening in the casing for the USB cable is positioned behind the hinge. To connect the USB cable the hinge needs to be turned fully to the inside of the strap. This safeguards that the USB cable cannot be connected while a person is wearing the DTI-2 on the wrist, which is a safety requirement. The Bluetooth transceiver (Panasonic PAN1325) is a complete module consisting of the electronics and ceramic antenna. The module is placed on the other end of the main printed circuit board. When the DTI-2 is strapped on a wrist in the prescribed position, this side of the main PCB is on the side of the wrist which is pointing away of the torso. Signal absorption by human tissue is minimized in this way.

The controller MSP430BT5190 from Texas Instruments is a dedicated type for use in combination with the PAN1325 transceiver and Mindtree software stack [12]. In terms of lowpower capabilities it is comparable to the rest of the MSP430 5series, and particularly comparable to the MSP430F5438A, which has excellent low-power capabilities, such as user defined frequencies, various sleep states, watchdog timer.

The data storage is done on a 2 GB microSD card, which can be replaced when necessary. An Alcor AU6431-JCF controller takes care of the communication via the USB cable, causing the card to mount as a drive on a computer, when the DTI-2 is connected.

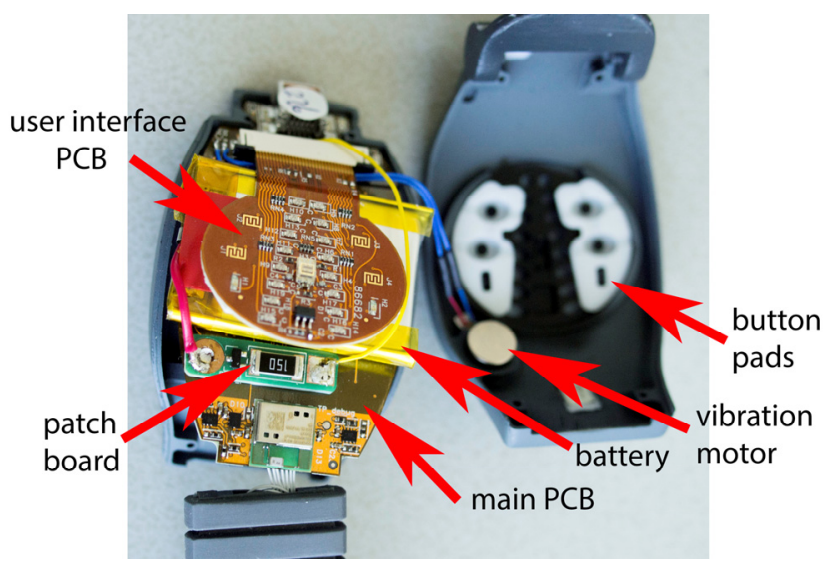

Figure 4 Assembly of the DTI-2 sensor wristband showing the user interface board, the patch board, the main PCB, the lithium battery, the vibration motor, and the button pads.

\subsubsection{Sensors}

The low power 3-axes accelerometer sensor type LIS331DLH made by STMicroelectronics is placed onto the main PCB.

The device temperature sensor AD7415 Analog Devices and the ambient light sensor TSL2561T from Taos are placed on the user interface PCB. A lens positioned above the sensor diodes of the ambient light sensor offers a wide angle of view from the top of the casing. By setting the integration time the right balance between sensitivity and power saving can be chosen. 
The sensor has two light sensitive diodes, one peaking in the red (at $640 \mathrm{~nm})$, and another in the near infra red $(940 \mathrm{~nm})$.

When a skin temperature sensor was to become part of the DTI2 bracelet it was decided to create a separate printed circuit board for the skin conductance sensor and the skin temperature sensor. An advantage of this configuration lies in the local filtering, amplification and digitization of the skin conductance signal. The pickup of electromagnetic noise can be minimized this way. Only digital signals at $3.3 \mathrm{~V}$ have to be communicated from the base of the wrist to the main printed circuit board.

The skin temperature sensor is an optical sensor. The medical grade Melexis 90615 SSG-DAA infrared thermometer was chosen, which offers a high 0.02 degree resolution combined with a low current consumption. The device can be put in a sleep mode to save energy. After resuming operation it takes $300 \mathrm{~ms}$ to obtain valid skin temperature data. Due to settling, in the first minute after putting the sensor on the wrist the temperature readouts are too high as can be seen in Figure 5. After this period the readings are accurate. The sensor is located on the underside of the wrist in-between the two skin conductance electrodes (see Figure 2).

The skin conductance sensor electronics uses a DC current through the skin. The skin contact electrodes for the skin conductance sensor are replaceable $0.95 \mathrm{~cm}$ diameter eyelets made by Micron products Inc., which are positioned at the underside of the wrist. In [11] the validity of this location as a position where emotion induced skin conductance signals can be measured is described.

A stable $1.2 \mathrm{~V}$ reference voltage causes a current to flow through the skin, a $1 \mathrm{k} \Omega$ safety resistor, and two $10 \mathrm{M} \Omega$ reference resistors. The voltage drop over the skin plus the safety resistor is amplified 2.8-fold, and filtered over a 2nd order low-pass Butterworth filter before analog to digital conversion by a 16-bit Analog Devices AD7685 ADC. The $\mathrm{ADC}$ uses a stable $3 \mathrm{~V}$ reference voltage.

In Figure 5 the result of 12 hours of data collection of the main sensors is shown in four graphs.

\subsubsection{User interface electronics}

The user interface of the DTI-2 consists of 4 buttons, two rows of eight LEDs plus some signaling LEDs. The buttons are meant for switching the device on/off, switching the Bluetooth radio on/off, setting an event marker, and showing the level of the battery or the level of the skin temperature or skin conductance.

\section{Software}

The architecture of the embedded software is organized in layers, as shown in Figure 6. The layers allow a separation of functionality, which facilitates local development, debugging and maintenance. The layers are decomposed in a number of software components, shown in Figure 7, each with a clear and upfront defined interface and function.

FreeRTOS is the selected operating system for the embedded software. Beside the Mindtree SDK tasks, also other tasks are created for scheduling the sampling and storing the data.
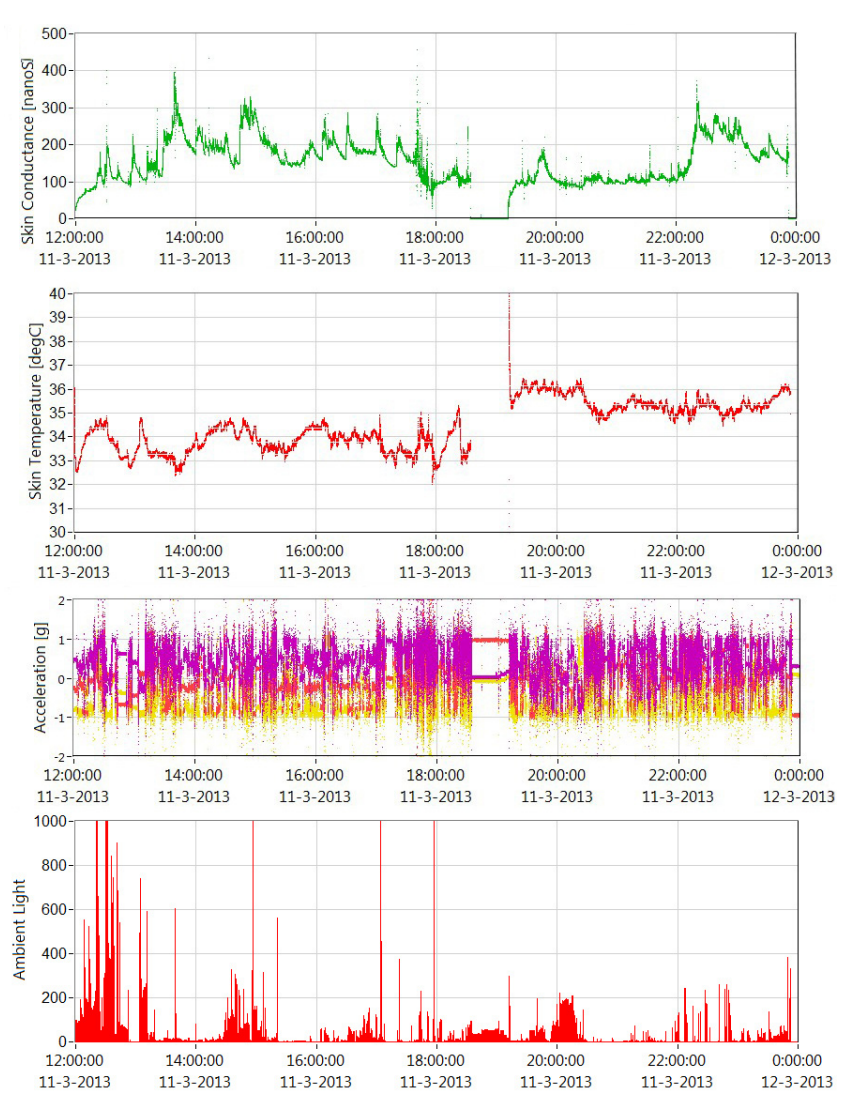

Figure 5 Sensor output of the DTI-2, showing a 12 hour

period. The top trace shows the skin conductance, the trace

below that shows the skin temperature, the third trace

visualizes the output of the motion sensor, and the bottom

trace the ambient light sensor readings. Around $19 \mathrm{~h} 00$ the

bracelet was taken off the wrist for about 30 minutes.

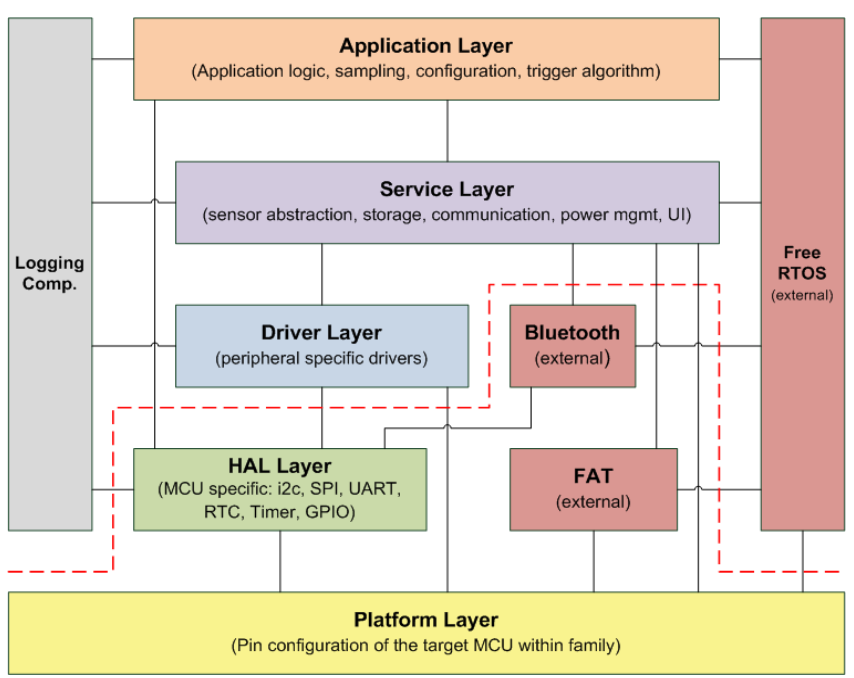

Figure 6 Layered software architecture 

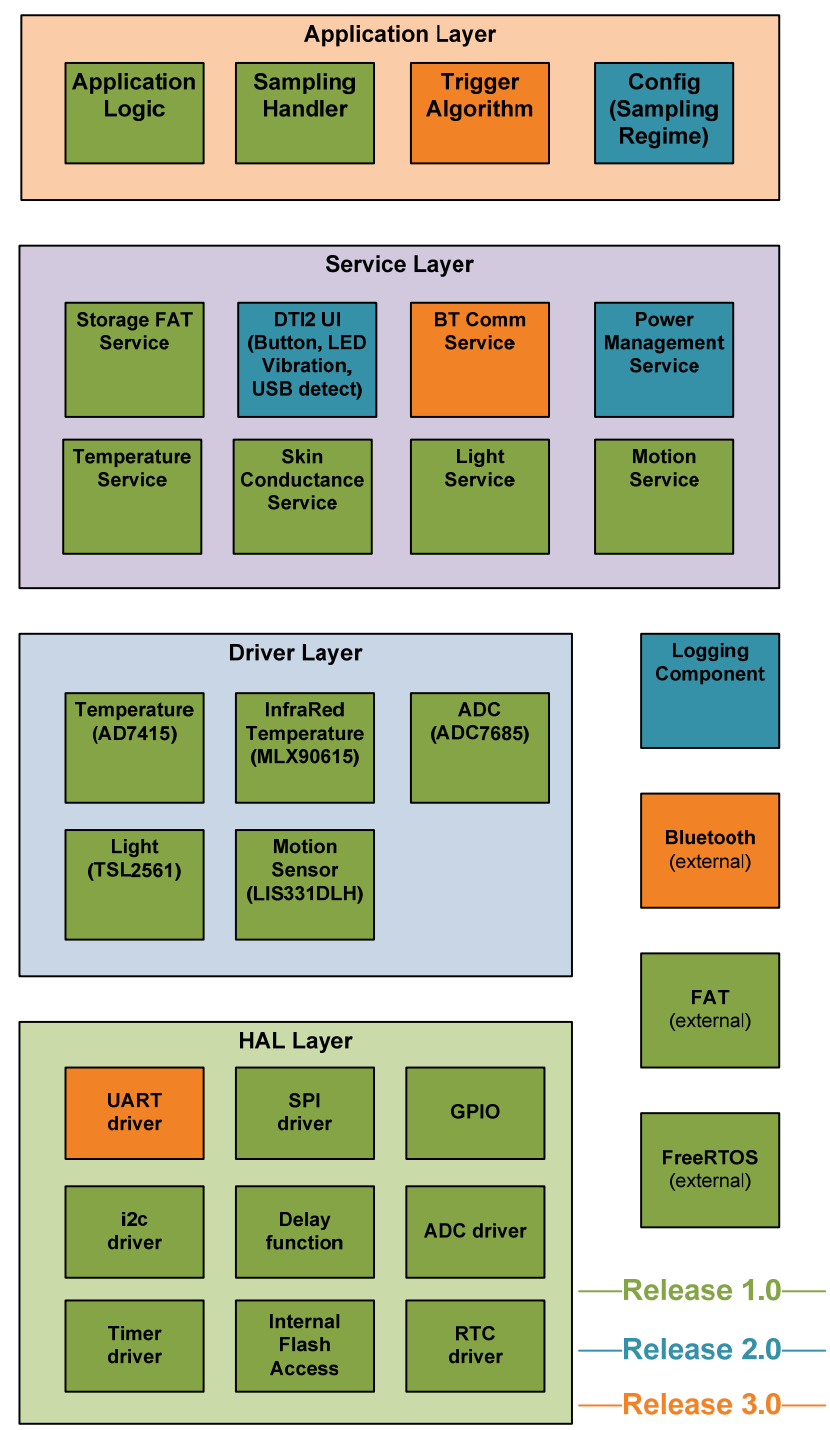

\section{Figure 7 Software components and their release number}

Product features (e.g. detection algorithms for emotional events, see [1]) and support for additional hardware sensors can be added by replacing or adding software components.

Requests from end users for adaptation of the software to the needs of their studies/trials are accommodated in the application layer. The service layer provides generic functional building blocks (e.g. temperature service, communication service) to the application layer. It also provides an abstraction of the lower layers, like sensor layer and the storage subsystem. The driver layer contains the sensor specific drivers. In this layer for example the driver for a specific make and model of a temperature sensor, but also drivers for the user interface components can be found. The HAL and platform layer contain the controller specific part of the embedded software like I2C and SPI and controller pin definitions. To port the software to another type of controller or even a PC-based platform, these layers have to be rewritten.

The Mindtree EtherMind Bluetooth SDK [12] is used to control MSP430BT5190 in combination with PAN1325 module. The Mindtree SDK uses FreeRTOS [13] as operating system. For
For storage of measurement data on the microSD card, a FAT32 file system is used. The file system contains the measurement data, logging data, and in some cases a settings file with a device configuration.

The software development process used an incremental approach. At each bi-weekly increment, well defined parts of the functionality have been delivered. Using this approach the software development could be tracked and steered in an agile approach. Each increment was finalized with a delivery.

A CR/PR system [14] is used for change control. New features of each increment and discovered problems are entered in this $\mathrm{CR} / \mathrm{PR}$ system. This system is linked directly to the software archive [14] to provide traceability through the entire development process.

For building and testing the intermediate software versions, the Jenkins continuous integration server [15] has been used. Jenkins is an open and widely used integration server and it was already successfully used in other projects. Using the integration server the embedded software build process has been automated. After each build, regression tests are run automatically.

The software development process is designed to accelerate future medical certification of the product software (e.g. CR/PR management, traceability, automated testing).

Parallel to the software development a separate power optimization track was started which is detailed in section 4 . After determining the optimum balance between power saving and other requirements the power optimizations will be integrated in the product software.

\section{POWER SAVING}

The DTI-2 is a wearable device, which is used in trials and studies. In these circumstances it is often desirable that the recharging of the battery occurs only once per week. With the product software described in section 3 a battery life of about 30 hours is reached. This was significantly lower than the target battery life of one week (see Table 1). It was undertaken to find out which software changes were needed to reach the target battery life, and what the consequences would be for flexibility in choosing the sampling rate, and the timestamp accuracy.

Based on the variations in the signal the optimal sampling rate for the various sensors can be determined (see Table 2). This is the lowest sampling rate where no useful information gets lost. For slow varying parameters, such as the device temperature a sampling rate of $0.1 \mathrm{~Hz}$ can be sufficient, whereas for the $3 \mathrm{D}$ accelerometer already at $10 \mathrm{~Hz}$ a small fraction of the motion information gets lost.

The DTI-2 uses the FreeRTOS to schedule all tasks, such as data sampling, data storage, wireless streaming, and user interface. In order to accommodate the different optimal sampling rates of the various sensors a separate task can be defined for each sensor. Such a task can also switch the sensor off when idle to save power, and to switch it on taking a warmup time into account to get an accurate reading. This offers a good data sampling rate flexibility of the system together with optimized power consumption.

This optimized scheme was implemented, and the current consumption measured. The results are shown in Table 2. The total average current consumption turned out to be lower when 
the device was not actively used, i.e. with the skin conductance electrodes not in skin contact. The active filter, which removes noise coming from 60 or $50 \mathrm{~Hz}$ mains uses about $0.5 \mathrm{~mA}$ extra current when the device is worn by a person.

Table 2 Contributions to average current consumption of power optimized scheme

\begin{tabular}{|l|c|c|}
\hline $\begin{array}{l}\text { Contribution to } \\
\text { average current } \\
\text { consumption }\end{array}$ & $\begin{array}{l}\text { Regular skin } \\
\text { conductance } \\
\mathbf{( 0 . 2} \boldsymbol{\mu S})\end{array}$ & $\begin{array}{l}\text { Device not in skin } \\
\text { contact (open } \\
\text { circuit) }\end{array}$ \\
\hline Idle task & $0.195 \mathrm{~mA}$ & $0.195 \mathrm{~mA}$ \\
\hline $\begin{array}{l}\text { Accelerometer @ 10 } \\
\mathrm{Hz}\end{array}$ & $0.258 \mathrm{~mA}$ & $0.258 \mathrm{~mA}$ \\
\hline $\begin{array}{l}\text { Device Temperature } \\
\text { sensor@0.2 Hz }\end{array}$ & $0.001 \mathrm{~mA}$ & $0.001 \mathrm{~mA}$ \\
\hline $\begin{array}{l}\text { Ambient light sensor } \\
@ 0.2 \mathrm{~Hz}\end{array}$ & $0.003 \mathrm{~mA}$ & $0.003 \mathrm{~mA}$ \\
\hline $\begin{array}{l}\text { Skin Temperature } \\
\text { sensor @0.2 Hz }\end{array}$ & $0.145 \mathrm{~mA}$ & $0.145 \mathrm{~mA}$ \\
\hline $\begin{array}{l}\text { Skin Conductance } \\
\text { sensor@ } 10 \mathrm{~Hz}\end{array}$ & $1.080 \mathrm{~mA}$ & $0.580 \mathrm{~mA}$ \\
\hline Data storage @ 10 Hz & $0.740 \mathrm{~mA}$ & $0.740 \mathrm{~mA}$ \\
\hline Total average current & $2.422 \mathrm{~mA}$ & $1.922 \mathrm{~mA}$ \\
\hline
\end{tabular}

Under usage conditions such as occur in studies and trials (i.e. continuous wearing the bracelet) the $2.4 \mathrm{~mA}$ average current combined with the $400 \mathrm{mAh}$ battery offers a over a week of battery life. The low C-rate (1/175) allows the extraction of about $425 \mathrm{mAh}$ from the battery. The design target of Table 1 is met.

The contributions of the various processes to the average current consumption can be visualized in a pie diagram, which is shown in Figure 8.

\subsection{Trade offs}

The problem using a RTOS with high number of tasks is that the execution of some of the tasks can be delayed, so the accurate timing of the data sampling is negatively affected.

In order to save RAM collected sensor data is stored in the flash memory of the MSP430, prior to batch writing it onto the microSD card. Erasing a section of the local flash takes $250 \mathrm{~ms}$ during which it cannot be used. This can cause a significant delay in storing the sensor data, causing an error of up to $250 \mathrm{~ms}$ in the timestamp.

Some of the end users need a very high time accuracy, for detecting the activity of the user with the accelerometer output for example. Then the accuracy of the timestamps is vital, and this kind of power saving has to be avoided

A solution to avoid multiple tasks is to do the data collection and storage in a single FreeRTOS task, as it is done in the product software described in section 3 . If the task is running with a timer at $100 \mathrm{~Hz}$ for example, sampling the accelerometer at $10 \mathrm{~Hz}$ is equivalent to skipping this step on the task 9 times out of 10. Changing the rate requires only to change the skip counter. Note that this requires the time between samples to be a multiple of $10 \mathrm{~ms}$. Using this approach, a complicated situation is created: for example starting one sensor at a certain time, wait $300 \mathrm{~ms}$, take the data, at the same time powering up another sensor at different moment to be sure that the simultaneous data collection does not occur (which may cause an unwanted delay). In the present firmware, the sensors are on all the time, causing the timing accuracy to be very good, but the power efficiency is poor.

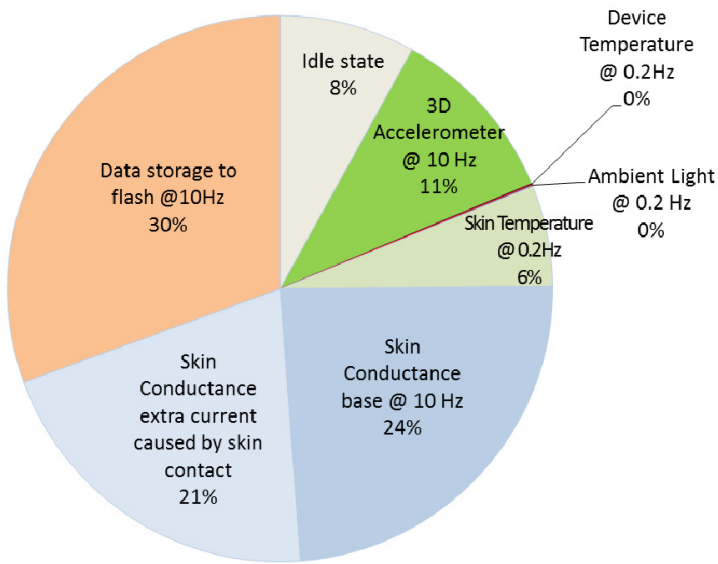

Figure 8 Pie diagram showing the contributions of the various components and processes to the total current consumption $(2.422 \mathrm{~mA})$ in the optimized low power mode

A solution that combines a high power efficiency and a good time accuracy is to do everything in one task, and each of the sensors is switched on at a precise moment, with always the same relative time lag with the other sensors. This is done in the task for temperatures and luminance in the power consumption optimized software. This, however, causes the sampling rate flexibility to decrease, as changing the sampling rate of one particular sensor is affecting the timing of all other events.

\section{Power efficiency}

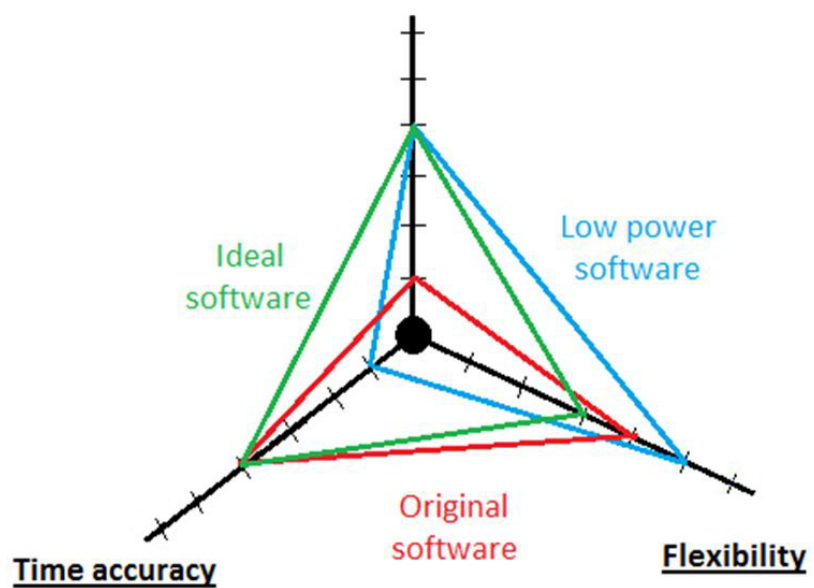

Figure 9 Time accuracy vs. Power efficiency vs. Flexibility. All parameters increase when moving away from the origin.

To have a combination of very good time accuracy, a good flexibility, and good power efficiency is impossible to achieve. Based on the end user needs the programmers of the application 
layer described in section 3 have to find a good compromise between these 3 parameters.

In general, it is probably better to reduce the sampling rate flexibility a bit, enabling the reduction of the average consumption of the production software. This will lead to a longer battery life. In Figure 9 the power optimized software solution is represented in blue (Low power software), the product software in red, and an ideal software in green. Tailored software solutions for different end-user needs, such as will be discussed in the next section, shall always be necessary.

\section{DISCUSSION}

The DTI-2 sensor bracelet is meant as an improvement of its predecessor DTI-1. In total 160 complete devices have been produced by Philips Innovation services (PInS). Whether the device is meeting expectations can only be judged from experiences in field use. The DTI-2 is approved to be used in a number of healthcare-related studies. One of these studies is the aggression prevention study Watch It!, aiming at early detection of imminent aggression in crisis wards of mental hospitals, and forensic psychiatry wards, and a follow up on the pilot study reported in [3].

In between the healthcare and lifestyle domain is the IET-ICT funded study led by the TU/e on burnout prevention of school teachers [9]. At the time of writing the pilot has been completed. It assured the feasibility of the test set-up, the enthusiasm of the pilot participants, and at least some relevancy of the gathered data. The full scale study on 30 persons is about to begin.

The sensor data is collected batch wise from the microSD cards and post processed as shown in Figure 10, which is reproduced from [9]. The end result of the processing are banded colors, representing more or less episodes with more or less tension, which are compared with the teaching roster of the participant.

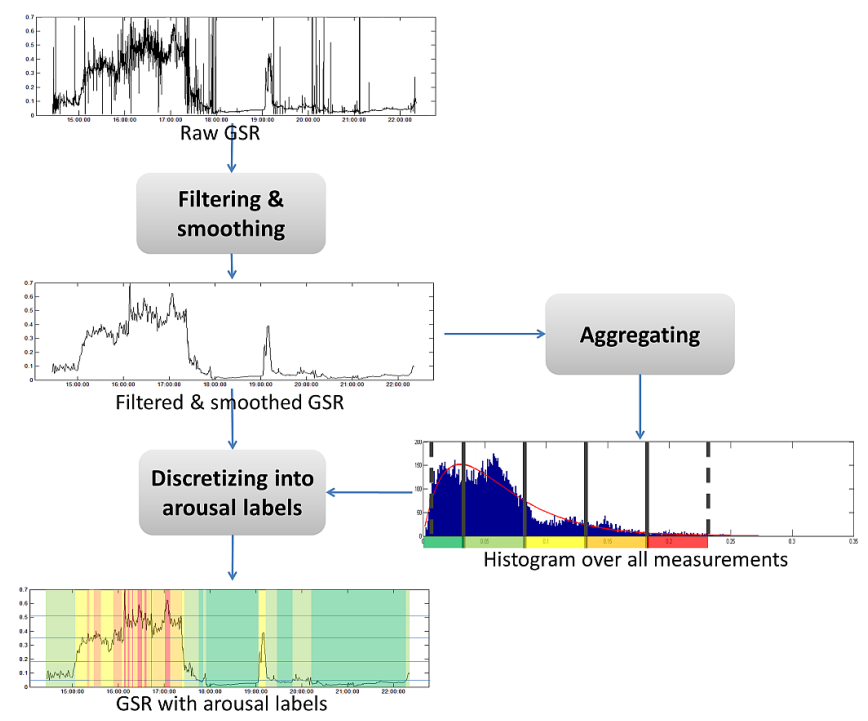

Figure 10 Data processing of DTI-2 data from teachers at risk of burnout obtained by the Stress@Work project [9].

More in the lifestyle domain is the IET-ICT funded study led by the Swedish Institute of Computer Science called WeCare [10], in which the emphasis is on communication of daily arousal data to the wearer of the DTI-2 bracelet via an Android app. There were some 30 participants, distributed over 3 groups: members of an elite orienteering team, office workers at Ericsson, female individuals between 25 and 55 years of age. Preliminary results indicate the people generally do accept the data as representing themselves, and on several occasions are the basis for enhanced awareness of their lifestyle. Most of the users were therefore motivated to keep on wearing the DTI-2 device for the duration of the test, even though they found that the DTI-2 did not look fancy at all. Some of them countered this disadvantage by (temporally) enhancing the DTI-2 with glue-on trinkets (see Figure 11).

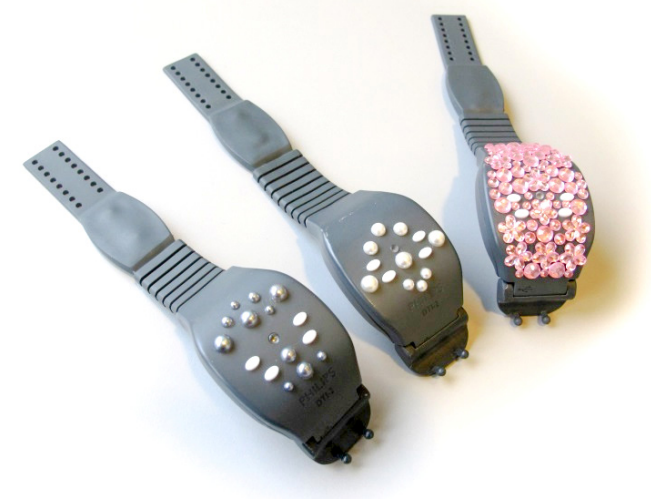

Figure 11 Example of DTI-2 appearance "enhancements" by participants

In Figure 12 an Android phone screenshot is shown of several hours of DTI-2 data that was streamed via the Bluetooth link. Higher arousal causes the coloration to shift towards warmer colors (orange/red), whereas lower arousal is depicted by cooler colors (green/blue). The spiral allows the visualization of a chosen section of history (in this case 6 hours). The user can tag emotional events, which show as pins in the spiral. The acceleration shows as a white jagged line on the inside of the spiral, with large peaks indicating vigorous motion.

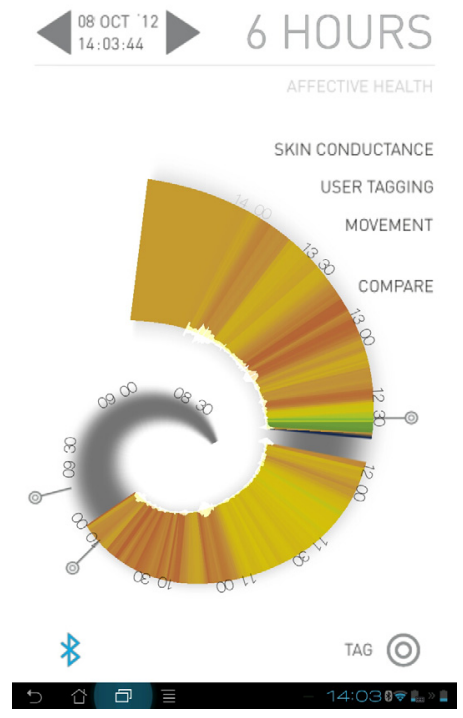

Figure 12 Affective Health User Interface, that runs as an app on an Android phone. It shows the skin conductance history as a colored spiral, and the motion sensor output as a jagged white edge of the spiral. User defined tags of emotional moments can be entered and show as pins on the spiral [10]. 
In the Affective Health study all data was streamed real time to the Android mobile phone of the participant. The power intensive use decreased battery life down to a single day. In Figure 13 an overview of more than 3 weeks of collected data for a participant is shown.
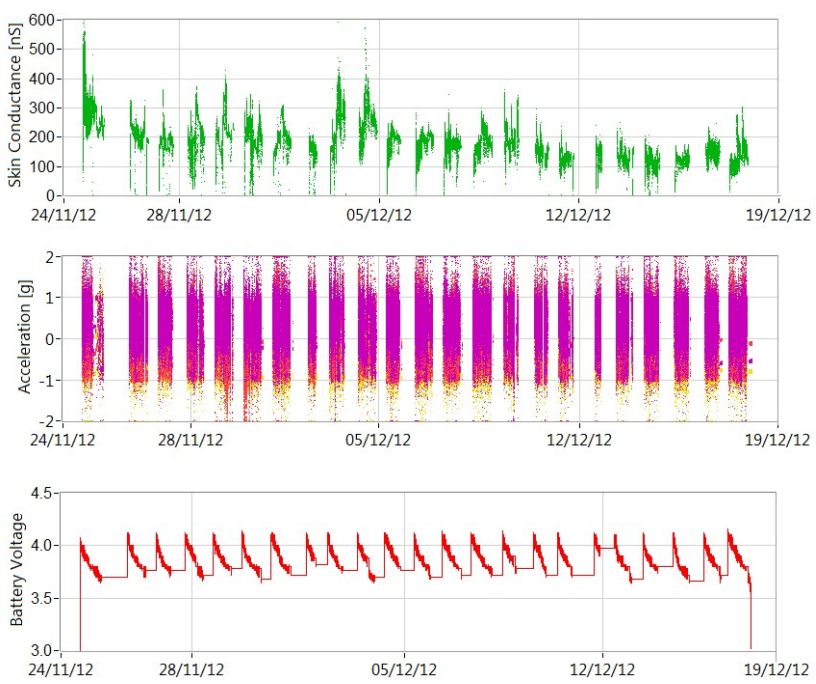

Figure 13 Over 3 weeks of data collected in the Affective Health study: the top trace shows the skin conductance, the middle trace the 3D accelerometry, and the bottom trace the battery voltage.

From the battery voltage trace it can be seen that never in the duration of the study a low battery condition $(>3 \mathrm{~V})$ was experienced. The choice to use Bluetooth for wireless data transfer was driven by the availability in mobile phones. A transition to Bluetooth low energy or ANT can be implemented as soon as the mobile phone software and hardware becomes available, by replacing the current Panasonic PAN1325A by a pin compatible PAN1323 module. This is expected to dramatically increase battery life during continuous wireless data streaming.

The DTI-2 bracelet continues to be used in trials and studies. The acceptance of skin conductance measured at the wrist is growing, also indicated by a mentioning of the DTI-1 in the recently revised standard book on skin conductance [16].

At the moment of writing the most promising option for the DTI-2 is the need for ambulatory sensors in psychiatry that can shed light on the mental state of crisis ward patients. [3].

\section{ACKNOWLEDGMENTS}

Our thanks to EIT ICT Labs who in part subsidized this work, to Richard van der Wolf of PInS for the electronics design, Tim Bakker for software writing and testing, Kris Godzwon for project management.

\section{REFERENCES}

[1] Westerink, J.H.D.M., Ouwerkerk, M., de Vries, J.J.G., De Waele, S., van den Eerenbeemd, J., van Boven, M. 2009. Emotion measurement platform for daily life situations. Proceedings Volume I, International Conference on Affective Computing \& Intelligent Interaction, ACII (Sep. 2009), Amsterdam, The Netherlands, 217-222.
[2] Ouwerkerk, M. 2011. Unobtrusive Emotions Sensing in Daily Life. Chapter 2 in: Westerink, J., Krans, M, Ouwerkerk, M. Sensing Emotions - The Impact of Context on Experience Measurements, Philips Research Book Series Volume 12, Springer, 21-40.

[3] Kuijpers, E., Nijman, H., Bongers, I. M. B., Lubberding, M., Ouwerkerk, M. 2012. Can mobile skin conductance assessments be helpful in signalling imminent inpatient aggression?. Acta Neuropsychiatrica, 24 (2012), 56-59.

[4] Westerink, J.H.D.M., van Beek, W.H.M., Daemen, E.M.L., Janssen, J.H., de Vries, J.J.G., Ouwerkerk, M., 2013. The Vitality bracelet: bringing balance to your life with psychophysiological measurements, , Fairclough, SH, Gilleade K (ed) Advances in Physiological Computing, Springer-Verlag London (in press).

[5] Djajadiningrat, T., Geurts, L., Munniksma, P.-R., Christiaansen, G., de Bont, J. 2009. Rationalizer: an emotion mirror for online traders. Proceedings of the 5th International Workshop on Design and Semantics of Form and Movement 39-48.

[6] Poh, Ming-Zher, Swenson, N.C., Picard, R.W., 2010. A Wearable Sensor for Unobtrusive, Long-Term Assessment of Electrodermal Activity. IEEE Transactions on Biomedical Engineering, 57, 5 (May 2010), 1243-1252.

[7] http://www.qsensortech.com

[8] van der Zwaag, M.D., Janssen, J.H., Westerink, J.H.D.M. 2013. Directing Physiology and Mood through Music: Validation of an Affective Music Player. IEEE Transactions on Affective Computing 4, 1 (2013) 57-68.

[9] Kocielnik, R., Sidorova, N., Maggi, F. M., Ouwerkerk, M., Westerink, J. H. D. M., 2013.Smart Technologies for Long-Term Stress Monitoring at Work, submitted to the26th IEEE International Symposium on ComputerBased Medical Systems.

[10] Sanches, P., Höök, K., Vaara, E., Weymann, C., Bylund, M., Ferreira, P., Peira, N., Sjölinder, M. 2010. Mind the body!: designing a mobile stress management application encouraging personal reflection. In Proceedings of the 8th ACM conference on designing interactive systems (2010). ACM, New York, NY, 47-56.

[11] van Dooren, M., de Vries, J.J.G., Janssen, J.H., Emotional sweating across the body: Comparing 16 different skin conductance measurement locations, Physiology \& Behavior, 106 (2012) 298-304.

[12] http://processors.wiki.ti.com/index.php?title=File:MSP430 BT5190_CC2560_Developers_Guide.pdf\&limit $=50$

[13] FreeRTOS: http://www.freertos.org/

[14] IBM Rational Synergy and Change suite http://www01.ibm.com/software/rational/products/synergychangesuite /

[15] Jenkins: http://jenkins-ci.org/

[16] Boucsein, W. 2012 Electrodermal Activity $2^{\text {nd }}$ Ed., Springer Science+ Business Media LLC, New York. (2012) 108-109.

[17] Lundqvist,L.-O., Carlsson, F.,Hilmersson, P., Juslin, P.N., Emotional responses to music: experience, expression, and physiology, Psychology of Music 37 (2009) 61-90. 